Pacific Journal of Mathematic 


\title{
ALTERNATING METHOD ON ARBITRARY RIEMANN SURFACES
}

\author{
LEO SARIO
}

1. Introduction. Schwarz gave the first rigorous construction of harmonic functions with given singularities on closed Riemann surfaces, by means of his alternating method for domains with annular intersection [16 ]. The method also is directly applicable to open Riemann surfaces of finite genus, since these can always be continued so as to form closed surfaces $[7 ; 8]$. For surfaces of infinite genus, this continuation is no longer possible. But if the surface is of parabolic type, Schwarz's method can still be used, a "null boundary" having no effect on the behaviour of the alternating functions [5; 11]. In the general case, there are two obstacles which prevent using Schwarz's method as such. First, if the surface has a large (ideal) boundary, the alternating functions are not determined by their values on the relative boundaries. Second, Schwarz's convergence proof fails, since the Poisson integral is inapplicable on arbitrary Riemann domains. We are going to show that, by certain changes of Schwarz's original method, these difficulties can be overcome.

This paper is a detailed exposition of a reasoning outlined in preliminary notes [9-11]. The manuscript of the paper was communicated (in French) to the Ifelsinki University in December, 1949. In the meanwhile, the author published a linear operator method [13], which also can be used to establish the results of these notes. A presentation of the classical alternating method for arbitrary Riemann surfaces seems, however, to have independent interest from a methodological viewpoint; such a presentation is the purpose of this paper.

The alternating method on Riemann surfaces, as sketched in [9-11], was referred to also in the recent papers of Kuramochi [1], Kuroda [2], Mori [3], and Ohtsuka [6]. A historical note on the method was given in [15].

2. Functions with vanishing conjugate $a_{0}$-periods. We start with two lemmas, which are basic for the alternating procedure.

Let $R$ be an arbitrary Riemann surface, and $G$ a subdomain, compact or not. The relative boundary $a_{0}$ of $G$, that is, the set of boundary points of $G$, interior 
to $R$, is assumed to consist of a finite number of closed analytic Jordan curves. On $a_{0}$, let $f$ be a real single-valued function, harmonic in an open set containing $a_{0}$.

LEMMA 1. There exists always a harmonic single-valued function $u$ in $G$ with the following properties:

$1^{\circ}$. $u$ takes on the values $f$ on $a_{0}$.

$2^{\circ}$. $u$ is bounded in $G$ and satisfies

$$
\min _{a_{0}} f \leq u \leq \max _{a_{0}} f
$$

$3^{\circ}$. u has a finite Dirichlet integral over $G$,

$$
D(u)=\iint_{G}|\operatorname{grad} u|^{2} d x d y<\infty .
$$

Here $z=x+$ iy is a local uniformizer of $R$.

$4^{\circ}$. The period along $a_{0}$ of the harmonic function $v$, conjugate to $u$, vanishes,

$$
\int_{a_{0}} d v=0
$$

Proof. If $G$ is compact, the lemma is evident. Suppose now $G$ is noncompact. We form an exhaustion $G_{1} \subset G_{2} \subset G_{3} \subset \ldots$ of $G$, such that the boundary of $G_{n}$ consists of $a_{0}$ and a set $a_{n}$ of closed analytic Jordan curves tending, for $n \longrightarrow \infty$, to the ideal boundary of $G$. Let $u_{n}$ be a harmonic function in $G_{n}$ which coincides with $f$ on $a_{0}$ and assumes on $a_{n}$ a constant value $c_{n}$. By Schwarz's reflexion principle, it is easy to see that $u_{n}$ is harmonic still on $a_{0}+a_{n}$.

We fix the constant $c_{n}$ as follows. We observe that $u_{n}$ depends continuously on $c_{n}$. The same is also true for the normal derivative $\partial u_{n} / \partial n$ on $a_{0}$ and, consequently, for

$$
\int_{a_{0}} d v_{n}=\int_{a_{0}} \frac{\partial v_{n}}{\partial s} d s=\int_{a_{0}} \frac{\partial u_{n}}{\partial n} d s
$$

where the meaning of $v_{n}$ and $d s$ is evident. If we choose

$$
c_{n}=\min _{a_{0}} f,
$$

then obviously $\partial u_{n} / \partial n \geq 0$, if $n$ denotes the interior normal of $a_{n}$ with respect to 
$G_{n}$. Hence, the period (4) is nonpositive in this case. If we had chosen

$$
c_{n}=\max _{a_{0}} f,
$$

we would have found by the same reasoning that the integral (4) is nonnegative. Thus, there must exist a value $c_{n}$ such that

$$
\min f \leq c_{n} \leq \max f,
$$

and such that the integral (4) vanishes. In the sequel we suppose that the constant $c_{n}$ has been selected according to this condition. We have then in $G_{n}$ the uniform estimate

$$
\min f \leq u_{n} \leq \max f
$$

In the sequence of the uniformly bounded functions $u_{n}$, there is a subsequence which converges uniformly in every closed subdomain of $G$ to a singlevalued function $u$, harmonic on $G+a_{0}$.

In order to see that $u$ is uniquely determined, we shall prove that the sequence $\left\{u_{n}\right\}$ itself, not only a subsequence, converges. Let $x_{n}$ be the harmonic function in $G_{n}$ with $x_{n}=0$ on $a_{0}, x_{n}=1$ on $a_{n}$. The sequence $\left\{x_{n}\right\}$ decreases monotonically, converging to a harmonic function $x$ on $G$ with $x=0$ on $a_{0}$. If $x \equiv 0, u$ is necessarily unique, since the difference $u^{\prime}-u^{\prime \prime}$ of two functions $u$ would assume, by

$$
\int d\left(v^{\prime}-v^{\prime \prime}\right)=0,
$$

both positive and negative values, and would be dominated by a multiple of $x$. llence we can confine our attention to the case $x \not \equiv 0$.

By Green's formula

$$
\int_{-a_{0}+a_{n}} x_{n} d \bar{v}_{n}-u_{n} d \bar{y}_{n}=0
$$

where $y_{n}$ is the harmonic conjugate of $x_{n}$, we have

$$
c_{n}=-\frac{\int_{a_{0}} f d \bar{y}_{n}}{\int_{a_{0}} d \bar{y}_{n}} .
$$

Hence $c_{n}$ converges to a unique constant $c$. Now let $z$ be an arbitrary (fixed) 
point of $G$. For sufficiently large $n, z$ is an interior point of $G_{n}$. Let $g_{n}$ be the Green's function of $G_{n}$ with the logarithmic pole at $z$. Draw a small circle $C$ about $z$. The Green's formula

$$
\int_{-a_{0}+a_{n}+C} u_{n} d \bar{h}_{n}-g_{n} d \bar{v}_{n}=0
$$

where $h_{n}$ is the harmonic conjugate of $g_{n}$, yields, if we let $C$ shrink to the point $z$,

$$
u_{n}(z)=\frac{1}{2 \pi}\left[\int_{a_{0}} f d h_{n}+c_{n}\left(2 \pi-\int_{a_{0}} d h_{n}\right)\right]
$$

This shows the convergence of $u_{n}(z)$ and thus uniqueness of $u$.

In order to prove that the function $u$ satisfies the conditions $1^{\circ}-3^{\circ}$, we note that the $u_{n}$ converge uniformly even on the closure of $C_{n}$. In fact, for $\epsilon>0$ and for $m, q$ sufficiently large, we have $\left(u_{m}-u_{q}\right)<\epsilon$ on $a_{n}$; and on $a_{0}$ this difference vanishes. By the maximum principle, the Cauchy criterion is fulfilled on the closure of $G_{n}$. In view of the harmonic boundary values and Schwarz's reflexion principle, the convergence is uniform even in a domain slightly extended beyond $a_{0}$ and $a_{n}$. From this we conclude that all derivatives of $u_{m}$ converge in the closure of $G_{n}$.

From the uniform convergence it follows that $u$ takes on the value $f$ on $a_{0}$. The condition $2^{\circ}$ is guaranteed by $(5)$. In order to study the condition $3^{\circ}$ we observe that, for $p>0$,

$$
D(u)=\lim _{n \rightarrow \infty} D_{n}(u)=\lim _{n \rightarrow \infty} \lim _{p \rightarrow \infty} D_{n}\left(u_{n+p}\right)
$$

where $D$ refers to $G$ and $D_{n}$ to $G_{n}$. We have

$$
D_{n}\left(u_{n+p}\right) \leq D_{n+p}\left(u_{n+p}\right)=\int_{-a_{0}+a_{n+p}} u_{n+p} d v_{n+p}
$$

In this expression, we have

$$
\int_{a_{n+p}} u_{n+p} d v_{n+p}=c_{n+p} \int_{a_{n+p}} d v_{n+p}=c_{n+p} \int_{a_{0}} d v_{n+p}=0
$$

Since the integral on the right in (9) extended over $a_{0}$ converges because of the uniform convergence of the $u_{n}$ and grad $v_{n}$, this integral is uniformly bounded. Hence, the condition $3^{\circ}$ is fulfilled.

The condition $4^{\circ}$ follows again by the uniform convergence of grad $v_{n}$ on $a_{0}$. 
This completes the proof of Lemma 1 .

3. Functions with nonvanishing conjugate $a_{0}$-periods. Suppose now that the region $G$ is not compact.

LEMMA 2. There exists always a single-valued harmonic function $u$ on $G$ which coincides with $f$ on $a_{0}$, and whose conjugate function $v$ has the period

$$
\int_{a_{0}} d v=1
$$

Proof. It suffices to consider the case $f \equiv 0$; in order to pass to the general case we have only to add to the constructed function a function furnished by Lemma 1.

Let now $u_{n}$ be a harmonic function in $G_{n}$ which vanishes on $a_{0}$ and assumes a constant value $d_{n}$ on $a_{n}$, such that the period of the conjugate function $v_{n}$ of $u_{n}$ is

$$
\int_{a_{0}} d v_{n}=1
$$

This choice is always possible, since the value of the foregoing integral is proportional to $d_{n}$. Obviously $u_{n}$ is a multiple of the harmonic measure of $a_{n}$.

By Green's formula

$$
\int_{-a_{0}+a_{n}}\left(u_{n+p} d v_{n}-u_{n} d v_{n+p}\right)=0
$$

we have

$$
\int_{a_{n}} u_{n+p} d v_{n}=d_{n}
$$

On the other hand, for the functions $u_{n+p}$, positive in $G_{n+1}$, we can use llarnack's principle, which can be expressed, in the present case, as follows. For all the functions $u_{n+p}$, there is a constant $M<\propto$ such that, on $a_{n}$, interior to $G_{n+1}$,

$$
\max _{a_{n}} u_{n+p}<M \min _{a_{n}} u_{n+p} .
$$

Hence, by (13) and

$$
\int_{a_{n}} d v_{n}=1
$$


we have

$$
\max _{a_{n}} u_{n+p}<M \int_{a_{n}} u_{n+p} d v_{n}=M d_{n}
$$

Thus, by the maximum principle, the functions $u_{n+p}$ are uniformly bounded in $G_{n}$ and form a compact family.

4. Oscillation of functions. In order to prove the convergence of the alternating functions, we still need a lemma concerning oscillations of functions.

Let $R$ be an arbitrary Riemann surface and $R_{0}$ a compact closed point-set on $R$. Consider all single-valued harmonic functions $u$ on $R$.

LEMMA 3. There exists a positive constant $q<1$, independent of $u$, such that for every $u$ the oscillations of $u$ on $R$ and $R_{0}$,

$$
\begin{aligned}
& S(u, R)=\sup _{R} u-\inf _{R} u \\
& S\left(u, R_{0}\right)=\max _{R_{0}} u-\min _{R_{0}} u,
\end{aligned}
$$

satisfy the inequality

$$
S\left(u, R_{0}\right) \leq q S(u, R) .
$$

Proof. For the two cases $S(u, R)=0$ and $S(u, R)=\infty$, the proposition (17) is evident; thus, it suffices to consider bounded nonconstant functions $u$. We normalize these functions, without loss of generality, by adding a constant and multiplying by a constant such that

$$
\sup _{R} u=1, \quad \underset{R}{\inf } u=0 .
$$

This being done, we have to prove the existence of a constant $q<1$ such that $S\left(u, R_{0}\right)<q$. If such a constant did not exist, there would be a sequence of functions $u_{1}, u_{2}, u_{3}, \ldots$ such that

$$
\lim _{n \rightarrow \infty} S\left(u_{n}, R_{0}\right)=1
$$

and, consequently,

$$
\max _{R_{0}} u_{n} \rightarrow 1, \quad \min _{R_{0}} u_{n} \rightarrow 0
$$


Among the functions $u_{n}$, uniformly bounded on $R$, one can select a subsequence, say again $\left\{u_{n}\right\}$, which tends uniformly to a function $u^{*}$, harmonic and singlevalued on $R$. The points $P_{n}$ and $Q_{n}$ where $u_{n}$ assumes maximum and minimum values, respectively, on the closed set $R_{0}$, accumulate at some points $P^{*}$ and $Q^{*}$ of $R_{0}$,

$$
P_{n} \rightarrow P^{*}, \quad Q_{n} \rightarrow Q^{*}
$$

It is easily seen that

$$
u^{*}\left(P^{*}\right)=1 \text { and } u^{*}\left(Q^{*}\right)=0 .
$$

In fact, if $u^{*}\left(P^{*}\right)$ were $<1$, let $\epsilon$ be a positive constant, $\epsilon<1 / 2\left(1-u^{*}\left(P^{*}\right)\right)$. By the continuity of $u^{*}$, there would be a neighborhood $K$ of $P^{*}$ such that, at each point $P$ of $K$,

$$
u^{*}(P)<u^{*}\left(P^{*}\right)+\epsilon \text {. }
$$

On the other hand, by the definition of $P_{n}$, for sufficiently large $n$,

$$
u_{n}\left(P_{n}\right)>1-\epsilon,
$$

and the points $P_{n}$ lie on $K$. Thus, at these points $P_{n}$, one would have

$$
u_{n}\left(P_{n}\right)-u^{*}\left(P_{n}\right)>1-u^{*}\left(P^{*}\right)-2 \epsilon=\text { const. }>0,
$$

in contradiction to the uniform convergence of the $u_{n}$ to $u^{*}$. This proves the first equality (22). The second one is proved in the same manner.

Consequently, the function $u^{*}$ would be harmonic, single-valued, and nonconstant on $R$, and would assume its maximum and minimum values at interior points of.$R$. This violation of the maximum principle disproves our antithesis. The lemma follows.

5. The existence theorem. After these preparations we are able to establish existence of the harmonic functions in question on the whole surface. Let $R_{0}$ be a subdomain of $R$ whose relative boundary, that is, the set of boundary points interior to $R$, consists of a finite set of closed analytic Jordan curves. The complement $G=R-R_{0}$ then consists of a finite number $m$ of disjoint domains $G_{i}(i=1,2, \cdots, m)$, compact or not. Let now $a_{i}$ be the common part of the boundaries of $R_{0}$ and $G_{i}$.

In each $G_{i}$, let $u_{i}$ be a given function, vanishing on $a_{i}$, harmonic, singlevalued and nonconstant in a neighborhood of $a_{i}$, having otherwise arbitrary singularities and, in case $G_{i}$ is noncompact, an arbitrary behaviour at the common 
(ideal) part of the boundaries of $R$ and $G_{i}$. Denote by $d s$ an arc element of $a_{i}$, and by $\partial u_{i} / \partial n$ the normal derivative of $u_{i}$ in the interior direction of $G_{i}$.

THEOREM. If $R_{0}$ is compact, then the condition

$$
\sum_{i=1} \int_{a_{i}} \frac{\partial u_{i}}{\partial n} d s=0
$$

guarantees the existence of a function $f$ on the whole surface $R$, satisfying the following conditions:

$1^{\circ}$. The function is harmonic, single-valued and nonconstant outside the possible singularities of the $u_{i}$.

$2^{\circ}$. The difference $f-u_{i}$ is harmonic, single-valued, and bounded in the whole region $G_{i}$, and has a finite Dirichlet integral over $G_{i}$.

In case $R_{0}$ is noncompact, the existence of $f$ satisfying $1^{\circ}$ and $2^{\circ}$ is always assured, independently of the condition (23). If this is satisfied, $f$ is bounded in $R_{0}$ and has there a finite Dirichlet integral.

Proof. Consider first the case where $R_{0}$ is compact. Let $R^{\prime}$ be another compact region $(\subset R)$, containing the closure of $R_{0}$ in its interior, and bounded by a finite number of closed analytic Jordan curves. The intersection $H_{i}=R^{\prime} \cap G_{i}$ is supposed to consist of one single region, bounded by $a_{i}$ and the intersection $b_{i}$ of $G_{i}$ and the boundary of $R^{\prime}$. Denote, for the time being, $u_{i}$ by $u_{i_{0}}$. In $R^{\prime}$, let $f_{0}$ be the harmonic function coinciding with $u_{i_{0}}$ on $b_{i}$. In $G_{i}$, form, by the procedure of Lemma 1 , a function $h_{i}$, harmonic and single-valued in $G_{i}$, coinciding with $f_{0}$ on $a_{i}$, bounded by the in equalities

$$
\min _{a_{i}} f_{0} \leq h_{i_{1}} \leq \max _{a_{i}} f_{0}
$$

possessing a finite Dirichlet integral over $G_{i}$,

$$
D_{i}\left(h_{i_{1}}\right)<\infty
$$

and satisfying the condition

$$
\int_{a_{i}} d k_{i_{1}}=0
$$

where $k_{i_{1}}$ is the harmonic conjugate of $h_{i_{1}}$. Write, in $G_{i}$,

$$
u_{i_{1}}=u_{i_{0}}+h_{i_{1}} \text {. }
$$


Let $f_{1}$ be the harmonic function in $R^{\prime}$ coinciding with $u_{i_{1}}$ on $b_{i}$. We then form again by the procedure of Lemma 1 a harmonic function $h_{i 2}$ in $G_{i}$ which assumes the values $f_{1}$ on $a_{i}$ and has the corresponding boundedness properties. We thus obtain successively a sequence of functions $h_{i n}$ and $u_{i n}$ in $G_{i}$, and $f_{n}$ in $R^{\prime}$, determined by the conditions

$$
\left\{\begin{array}{l}
f_{n}=u_{i n} \text { on } b_{i}, \\
h_{i(n+1)}=f_{n} \text { on } a_{i} \\
u_{i(n+1)}=u_{i 0}+h_{i(n+1)} \text { in } G_{i},
\end{array} \quad(n=0,1,2, \cdots)\right.
$$

and having the properties

$$
\begin{gathered}
\min _{a_{i}} f_{n} \leq h_{i(n+1)} \leq \max _{a_{i}} f_{n}, \\
D_{i}\left(h_{i n}\right)<\infty, \\
\int_{a_{i}} d k_{i n}=0,
\end{gathered}
$$

where $k_{i n}$ is the conjugate function of $h_{i n}$.

One has to prove the convergence of the functions $f_{n}$ and $u_{i n}$ toward a desired common function $f$. We shall show first the convergence of the functions $f_{n}$ on the closure $\bar{R}^{\prime}$ of $R^{\prime}$.

By Cauchy's criterion, this convergence is assured as soon as the difference $f_{n+p}-f_{n}$ tends, for $n, p \rightarrow \infty$, toward zero on the boundary $b$ of $R^{\prime}$. In order to use Lemma 3, we shall reduce estimation of this difference to that of its oscillation on $b$,

$$
\left|f_{n+p}-f_{n}\right| \leq S\left(f_{n+p}-f_{n} ; b\right)
$$

this inequality being valid as soon as

$$
\min _{R^{\prime}}\left|f_{n+p}-f_{n}\right|=0
$$

We shall now prove the latter relation.

Let $x_{i}$ be, in $H_{i}=R^{\prime} \cap G_{i}$, the harmonic function vanishing on $a_{i}$ and assuming the constant value 1 on $b_{i}$. Let $y_{i}$ be the conjugate function of $x_{i}$. The condition $(30)$ is satisfied if 


$$
\sum_{i} \int_{b_{i}}\left(f_{n+p}-f_{n}\right) d y_{i}=0
$$

In order to establish this equation, we make use of Green's formula

$$
\int_{-a_{i}+b_{i}}\left(f_{n} d y_{i}-x_{i} d g_{n}\right)=0
$$

where $g_{n}$ is the harmonic conjugate of $f_{n}$. It follows, by

$$
\sum \int_{b_{i}} d g_{n}=0
$$

that

$$
\sum \int_{a_{i}} f_{n} d y_{i}=\sum \int_{b_{i}} f_{n} d y_{i}
$$

On the other hand, the formula

$$
\int_{-a_{i}+b_{i}}\left(u_{i(n+1)} d y_{i}-x_{i} d v_{i(n+1)}\right)=0
$$

gives, in view of (23), ( 28$)$, and, accordingly, of

$$
\sum \int_{b_{i}} d v_{i(n+1)}=\sum \int_{b_{i}} d v_{i_{0}}=0
$$

the relation

$$
\sum \int_{a_{i}} u_{i(n+1)} d y_{i}=\sum \int_{b_{i}} u_{i(n+1)} d y_{i}
$$

By (25), (32), and (33), we have

$$
\sum \int_{b_{i}} f_{n} d y_{i}=\sum \int_{b_{i}} f_{n+1} d y_{i}
$$

This yields the desired equality ( 31 ).

The problem of convergence of $f_{n}$ has herewith been reduced to the estimation of the oscillation $S\left(f_{n+p}-f_{n} ; b\right)$. We have first 


$$
S\left(f_{n+p}-f_{n} ; b\right) \leq \sum_{m=1}^{p} S\left(f_{n+m}-f_{n+m-1} ; b\right) .
$$

To estimate $S\left(f_{n+1}-f_{n} ; b\right)$, note first that

$$
\begin{aligned}
& f_{n+1}-f_{n}=h_{i(n+1)}-h_{i n} \text { on } b_{i}, \\
& f_{n}-f_{n-1}=h_{i(n+1)}-h_{i n} \text { on } a_{i} .
\end{aligned}
$$

Since the functions $h_{i(n+1)}$ and $h_{i n}$ were constructed by the procedure of Lemma 1 as limits of certain harmonic functions coinciding with $f_{n}$ and $f_{n-1}$, respectively, on $a_{i}$, and satisfying the condition (26), the difference $h_{i(n+1)}-h_{i n}$ can be considered as defined by the procedure of Lemma 1 , with the boundary values $f_{n}-f_{n-1}$ on $a_{i}$. Thus, this difference satisfies the corresponding condition in the whole $G_{i}$ :

$$
\min _{a_{i}}\left(f_{n}-f_{n-1}\right) \leq h_{i(n+1)}-h_{i n} \leq \max _{a_{i}}\left(f_{n}-f_{n-1}\right)
$$

The relations $(36)-(38)$ yield

$$
S\left(f_{n+1}-f_{n} ; b\right) \leq S\left(f_{n}-f_{n-1} ; a\right),
$$

where $a$ is the boundary of $R_{0}$.

On the other hand, by Lemma 3 , applied to the difference $f_{n}-f_{n-1}$, the domain $R^{\prime}$, and the boundary of $R_{0}$, we have

$$
S\left(f_{n}-f_{n-1} ; a\right) \leq q \cdot S\left(f_{n}-f_{n-1} ; b\right),
$$

$q$ being a positive constant $<1$. Thus,

$$
S\left(f_{n+1}-f_{n} ; b\right) \leq q S\left(f_{n}-f_{n-1} ; b\right) .
$$

By repetition of the same reasoning starting from $f_{n}-f_{n-1}$, and so on, we obtain the desired estimate

$$
S\left(f_{n+1}-f_{n} ; b\right) \leq q^{n} S_{0},
$$

where $S_{0}$ signifies the constant $S\left(f_{1}-f_{0} ; b\right)$.

Applied to (35), this yields

$$
S\left(f_{n+p}-f_{n} ; b\right)<q^{n} \frac{S_{0}}{1-q} .
$$


The right side tends to zero, independently of $p$. By (29), Cauchy's criterion is satisfied and the uniform convergence of the functions $f_{n}$ to a single-valued harmonic function $f$ in $R^{\prime}$ has been proved.

The convergence of the functions $h_{i n}$ follows immediately. In fact, the relation ( 38 ), applied to the difference $h_{i(n+p)}-h_{i n}$, gives

$$
\max _{G_{i}}\left|h_{i(n+p)}-h_{i n}\right| \leq \max _{a}\left|f_{n+p-1}-f_{n-1}\right| \text {. }
$$

This implies, by the convergence of $f_{n}$, that of $h_{i n}$. The limit function $h_{i}$ is harmonic and single-valued in $G_{i}$. The corresponding limit of the functions $u_{i n}$ is $u_{i}+h_{i}$, where we use again $u_{i}$ instead of $u_{i 0}$.

The functions $f$ and $u_{i}+h_{i}$ are identical in $H_{i}=R^{\prime} \cap G_{i}$. In fact, the difference $f_{n}-u_{i n}$ vanishes on $b_{i}$ and coincides with $f_{n}-f_{n-1}$ on $a_{i}$, thus tending to zero on $a_{i}+b_{i}$ and hence on $H_{i}$.

Denote in the sequel by $f$ the function thus obtained on the whole surface $R$. It remains to show that it satisfies the conditions $1^{\circ}-2^{\circ}$ of the theorem.

Since the difference $f-u_{i}=h_{i}$ is harmonic and single-valued in $G_{i}$, the same is true of the function $f$ except at the singularities of $u_{i}$. We shall show that $f$ does not reduce to a constant.

Let $h_{i}^{\prime}$ be the harmonic function in $G_{i}$, constructed by the procedure of Lemma 1 to coincide with $h_{i}=f-u_{i}$ on $a_{i}$. Then $h_{i}^{\prime}-h_{i n}$ is the function in $G_{i}$ corresponding, by this procedure, to the values $h_{i}^{\prime}-h_{i n}$ on $a_{i}$. By the relations

$$
\min _{a_{i}}\left(h_{i}-h_{i n}\right) \leq h_{i}^{\prime}-h_{i n} \leq \max _{a_{i}}\left(h_{i}-h_{i n}\right),
$$

valid in $G_{i}$, and by the convergence $h_{i n} \longrightarrow h_{i}$ on $a_{i}$, the functions $h_{i n}$ converge uniformly to $h_{i}^{\prime}$ in $G_{i}$; that is, $h_{i}^{\prime} \equiv h_{i}$. By Lemma 1 , this implies that

$$
\min _{a_{i}} f \leq h_{i} \leq \max _{a_{i}} f
$$

If now $f$ were constant, the same would be the case with $h_{i}$, hence also with $u_{i}=$ $f-h_{i}$, contrary to our assumptions. This proves the property $1^{\circ}$.

Property $2^{\circ}$ follows from the fact just mentioned that $h_{i}=f-u_{i}$ is a harmonic function in $G_{i}$ constructed by the procedure of Lemma 1. This completes the proof of the theorem for the case where $R_{0}$ is compact.

The case where $R_{0}$ is noncompact reduces simply to the preceding case. We only have to isolate the common part of the boundaries of $R_{0}$ and $R$ from $R-R_{0}$ 
by a finite set $a_{0}$ of simple analytic Jordan curves which divide $R_{0}$ into a compact domain $R_{0}^{*}$ and a noncompact domain $G_{0}$. By Lemmas 1 and 2, there exists in $G_{0}$ a function $u_{0}$, harmonic and single-valued, vanishing on $a_{0}$ and having a prescribed period for the conjugate function $v_{0}$. We select this period in accordance with the condition

$$
\int_{a_{0}} d v_{0}=-\sum_{1}^{m} \int_{a_{i}} d v_{i}
$$

All the assumptions of the first part of our theorem are thus satisfied. Hience, there exists a function $f$ on $R$ which fulfills the conditions stated in the latter part of our theorem.

6. Applications. The theorem thus proved has applications to the classification of Riemann surfaces and to the theory of Abelian integrals as announced in $[10 ; 11]$. Here we confine our attention to some typical corollaries.

COROLLARY l. There are Green's functions on a Riemann surface $R$ if and only if the boundary has a positive harmonic measure.

Proof. Suppose there is a Green's function $g$ on $R$. Let $P: z=0$ be its logarithmic pole, in a parameter $\operatorname{disc} K:|z| \leq 1$. In $G_{1}=R-\bar{K}$, let $u$ be the harmonic function constructed by the procedure of Lemma 1 for values $u=g$ on $a_{1}:|z|=1$. Then $g-u$ is bounded in $G_{1}$,

$$
|g-u| \leq M<\infty,
$$

and has a nonvanishing conjugate period. This clearly implies the existence of a nonvanishing harmonic measure $\omega$ in $G_{1}$.

Conversely, suppose $\omega \neq 0$ in $G_{1}$. Multiply $\omega$ by such a constant that the conjugate of the function $u_{1}$ thus obtained has the period $2 \pi$ along $a_{1}$. Take as the domain $R_{0}$ of our theorem the annulus $1 / 2<|z|<1$. In $G_{2}:|z|<1 / 2$ write $u_{2}=$ $\log 1 /|z|$. By our theorem, there is a function $g^{\prime}$ on $R$ with the pole $\log 1 /|z|$ at $z=0$ and such that $g^{\prime}-u_{1}$ is bounded in $G$. The existence of a Green's function follows.

This result [10], proved later also by Virtanen [18] and Kuroda [2], shows that the classification of Riemann surfaces in those with "null-boundary" and “positive boundary" coincides with Riemann's classification on the basis of existence or nonexistence of Green's functions.

Another application of our theorem is a criterion for the existence of single- 
valued nonconstant harmonic functions which are bounded ( $H B$ ) or have a finite Dirichlet integral (IID). It was stated by Nevanlinna [4] and Virtanen [17] that there are functions HB or HD on $R$ if and only if $R$ has a null-boundary. This assertion has been disproved by Ahlfors and Royden. A correct criterion follows:

COROLLARY 2. There are functions HB or HD on a given Riemann surface $R$ if and only if some function $u$ of class $\mathrm{HB}$ or $\mathrm{HD}$ respectively in $G$ satisfies the conditions $u=0$ on $a, \int_{a} d v=0$.

The condition of a positive harmonic measure is equal to the first condition given above [8]. Thus, the inadequacy of Nevanlinna's statement is due to the lack of the second condition.

A further application deals with Abelian integrals. The following problem was stated by Myrberg in 1948 (October 13, at Helsinki University): Does there exist a nonconstant harmonic function with a finite Dirichlet integral on an arbitrary open Riemann surface $R$. The above theorem gives $[11]$ :

COROLLARY 3. On an arbitrary Riemann surface of positive genus there exist Abelian integrals of the first, second, and third kind which possess a finite Dirichlet integral outside a neighborhood of the singularities.

The Abelian integrals, the existence of which was thus proved, have later been investigated by Virtanen and Nevanlinna. The existence proof can also be performed by adapting the classical reasoning of Weyl.

Another immediate consequence of the foregoing theorem is the following result, proved first by Nevanlinna [5] using integral equations. Let $R$ be an open Riemann surface of parabolic type, and let $A$ and $B$ be two noncompact subdomains such that $A \cap B$ is a doubly connected region, bounded by two analytic Jordan curves. Let $a$ and $b$ be two single-valued harmonic functions in $A \cap B$.

COROLLARY 4. If the difference of the conjugate functions of $a$ and $b$ is single-valued, then there exists a harmonic function $f$ on $R$ such that $f-a$ in $A$, $f-b$ in $B$, are harmonically continuable, single-valued, and bounded.

To prove this, we have only to select as the domain $R_{0}$ of our theorem a region interior to $A \cap B$, separating the two boundary curves of the latter, and the existence of $f$ is assured.

In several related problems, an extremal method [14] seems to be more powerful than the alternating methods. A comparative survey on these methods was given in [ 15 ]. 


\section{REFERENCES}

1. Zenjiro Kuramochi, Potential theory and its applications, I, Osaka Math. J. 3 (1951) 123-174.

2. Tadashi Kuroda, Some remarks on an open Riemann surface with null boundary, Tohoku Math. J. (2) 3(1951), 182-186.

3. Akira Mori, On the existence of harmonic functions on a Riemann surface, J. Fac. Sci. Univ. Tokyo, Sect. I, 6 (1951), 247-257.

4. Rolf Nevanlinna, Quadratisch integrierbare Differentiale auf einer Riemannschen Mannigfaltigkeit, Ann. Acad. Sci. Fennicae A. I. 1 (1941), 1-34.

5. - Über die Neumannsche Methode zur Konstruktion von Abelschen Integralen, Comment. Math. Helv. 22 (1949), 302-316.

6. Makoto Ohtsuka, Dirichlet problems on Riemann surfaces and conformal mappings, Nagoya Math. J. 3 (1951), 91-137.

7. Leo Sario, Über Riemannsche Flächen mit hebbarem Rand, Ann. Acad. Sci. Fennicae A.I. 50 (1948), 1- 79.

8. Trondheim 1949 (1952), 229-238.

9. - Existence des fonctions d'allure donnée sur une surface de Riemann arbitraire, C.R. Acad. Sci. Paris 229 (1949), 1293-1295.

10. - Quelques propriétés á la frontière se rattachant à la classification des surfaces de Riemann, C.R. Acad. Sci. Paris 230 (1950), 42-44.

11. Existence des intégrales abéliennes sur les surfaces de Riemann arbitraires, C.R. Acad. Sci. Paris 230 (1950), 168-170.

12. - Alternating method for analytic functions, Bull. Amer. Math. Soc. 57 (1951), 275-276.

13. - A linear operator method on arbitrary Riemann surfaces, Trans. Amer. Math. Soc. 72(1952), 281-295.

14. - An extremal method on arbitrary Riemann surfaces, Trans. Amer. Math. Soc. 73 (1952), 459-470.

15. - Construction of functions with prescribed properties on Riemann surfaces, Conference on Riemann Surfaces in Commemoration of the Hundredth Anniversary of Riemann's; Dissertation, Princeton, 1951, Ann. of Math. Studies ( to appear).

16. H. A. Schwarz, Ges. Math. Abh., Berlin 1890, vol. II, pp. 133-171, 303-306, 356$358,360-362$.

17. K. I. Virtanen, Über Abelsche Integrale auf nullberandeten Riemannschen Flächen von unendlichem Geschlecht, Ann. Acad. Sci. Fennicae A.I. 56 (1949), 1-44.

18. - Über die Existenz von beschränkten harmonischen Funktionen auf offenen Riemannschen Flächen, Ann. Acad. Sci. Fennicae A.I. 75 (1950), 1-7.

\section{STANFORd UniverSITY}





\section{PACIFIC JOURNAL OF MATHEMATICS}

\section{EDITORS}

\author{
R. M. BOEINSON \\ University of California \\ Berkeley 4, California \\ E. HewitT \\ University of Washington \\ Seattle 5 , Washington
}

\section{R. P. DILWOR TH}

California Institute of Technology

Pasadena 4, California

E. F. BECKENBACH

University of California

Los Angeles 24, California

\section{ASSOCIATE EDITORS}

$\begin{array}{ll}\text { H. BUSEMANN } & \text { P. R. HALMOS } \\ \text { HERBERT FFDERER } & \text { IIEINZ HOPF } \\ \text { MARSHALL IJALL } & \text { R. D. JAMES }\end{array}$

\author{
BøRGE JESSEN \\ PAUL LÉVY \\ GEORGE PÓLYA
}

\author{
J. J. STOKER \\ E. G. STR AUS \\ KÔSAKU YOSIDA
}

\section{SFONSORS}

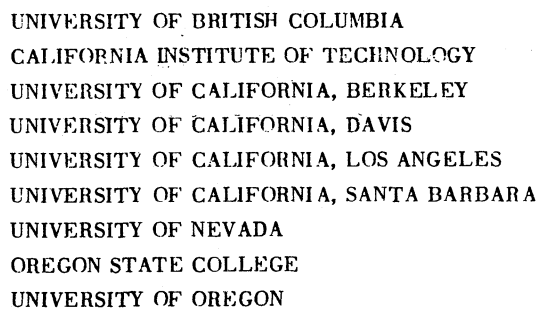

\author{
UNIVERSITY OF SOUTHERN CALIFORNIA \\ STANFORD RESEARCH INSTITUTE \\ STANFORD UNIVERSITY \\ WASHINGTON STATE COLLEGE \\ UNIVERSITY OF WASHINGTON \\ AMERICAN MATHEMATICAL SOCIETY \\ NATIONAL BUREAU OF STANDARDS, \\ INSTITUTE FOR NUMERICAL ANALYSIS
}

Mathematical papers intended for publication in the Pacific Journal of Mathematics should be typewritten (double spaced), and the author should keep a complete copy. Manuscripts may be sent to any of the editors except Robinson, whose term expires with the completion of the present volume; they might also be sent to M.M. Schiffer, Stanford University, Stanford, California, who is succeeding Robinson. All other communications to the editors should be addressed to the managing editor, E. F. Beckenbach, at the address given above.

Authors are entitled to receive 100 free reprints of their published papers and may obtain additional copies at cost.

The Pacific Journal of Mathematics is published quarterly, in March, June, September, and December. The price per volume (4 numbers) is $\$ 8.00$; single issues, $\$ 2.50$. Special price to individual faculty members of supporting institutions and to individual members of the American Mathematical Society: $\$ 4.00$ per volume; single issues, $\$ 1.25$.

Subscriptions, orders for back numbers, and changes of address should be sent to the publishers, University of California Press, Berkeley 4, California.

Printed at Ann Arbor, Michigan. Entered as second class matter at the Post Office, Berkeley, California.

\section{UNIVERSITY OF CALIFORNIA PRESS • BERKELEY AND LOS ANGELES}




\section{Pacific Journal of Mathematics}

\section{Vol. 3, No. 3 \\ May, 1953}

L. Carlitz, Some theorems on generalized Dedekind sums ............ 513

L. Carlitz, The reciprocity theorem for Dedekind sums ............. 523

Edward Richard Fadell, Identifications in singular homology theory..... . . 529

Harley M. Flanders, A method of general linear frames in Riemannian

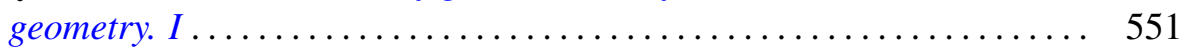

Watson Bryan Fulks, The Neumann problem for the heat equation ........ 567

Paul R. Garabedian, Orthogonal harmonic polynomials.............. 585

R. E. Greenwood and Andrew Mattei Gleason, Distribution of round-off errors for running averages .............................. 605

Arthur Eugene Livingston, The space $H^{p}, 0<p<1$, is not normable ... 613

M. N. Mikhail, On the order of the reciprocal set of a basic set of polynomials .......................................... 617

Louis Joel Mordell, On the linear independence of algebraic numbers . . . . 625

Leo Sario, Alternating method on arbitrary Riemann surfaces .......... 631

Harold Nathaniel Shapiro, Iterates of arithmetic functions and a property of the sequence of primes.............................. 647

H. Shniad, Convexity properties of integral means of analytic functions . . . . 657

Marlow C. Sholander, Plane geometries from convex plates ........... 667 